Lymphology 52 (2019) 166-176

\title{
EFFICACY OF KINESIO TAPING IN EARLY STAGE BREAST CANCER ASSOCIATED LYMPHIEDEMA: A RANDOMIZED SINGLE BLINDED STUDY
}

\author{
T. Ozsoy-Unubol, C. Sanal-Toprak, Y. Bahar-Ozdemir, G. Akyuz
}

Department of Physical Medicine and Rehabilitation, Marmara University School of Medicine, Istanbul, Turkey

\section{ABSTRACT}

This study was designed to evaluate the effectiveness of kinesio taping compared to compression garment in treatment of early stage breast cancer-associated lymphedema (BCAL). Patients between 18-70-years old who had unilateral stage 1 BCAL were randomized into group I kinesio taping (KT) and group II compression garment (CG) for this single blinded study. KT was applied with a lymphatic correction technique in three-four day intervals for four weeks. At the end of the fourth week, patients were suggested to wear CGs. Patients in group II were treated daily for 23-hours in CGs. Education, preventive measures, and exercises were given to both groups. All patients were evaluated before the treatment (T0), immediate post treatment (T1), and three months after treatment (T2). Circumference differences were measured between the extremities with a nonelastic tape at five levels. Additionally, shoulder range of motion (ROM) was measured, and pain, heaviness, and sensation of tightness were evaluated with a visual analog scale (VAS). Thirty-five patients with stage 1 $B C A L$ were included and randomized to the $K T(n=16)$ and CG $(n=19)$ groups. Demographic data and baseline clinical characteristics were similar. Both groups had reductions in all levels of arm circumference differences at immediate post-treatment and three months after treatment. Pain, tightness, and heaviness scores significantly decreased for both groups at immediate post-treatment and third month. Patients in the KT group had significantly lower pain sores than patients in the CG group. Results demonstrated that both modalities had similar effects in the treatment of early stage $B C A L$. For patients with early stage BCAL, $K T$ can be an alternative treatment to $C G$ for patients who have difficulties in obtaining and wearing CGs.

Keywords: compression garment, lymphedema, breast cancer, kinesio taping, randomized clinical trial

Breast cancer-associated lymphedema (BCAL) is one of the most common presentations of upper extremity lymphedema (LE), which results from obstructions to lymphatic vessels and/or lymph nodes by the tumor itself or due to treatment procedures (e.g., surgery or radiation). The incidence of unilateral arm LE ranges from $16.6 \%$ to $21.4 \%$ in breast cancer survivors (1) with incidence as high as $58.4 \%$ in patients who had been treated with neoadjuvant chemotherapy and axillary dissection (2). Risk factors for the development of LE include extensive surgery (e.g., axillary lymph-node dissection, dissection of multiple lymph nodes, and mastectomies), obesity, adjuvant taxanes, radiation therapy, and 
infection $(3,4)$. The signs and symptoms of LE include upper extremity swelling, pain, tightness, heaviness, discomfort, limited arm range of motion, and skin changes (5). According to volume or circumference differences between two extremities, staging gradually increases from subclinical LE (stage 0) to severe LE (stage 3). The International Society of Lymphology (ISL) states that stage 0 refers to a latent or subclinical condition where swelling is not evident. According to this staging, early accumulation of fluid in stage 1 is reversible, limb elevation rarely reduces swelling in stage 2, and fibrosis and trophic skin changes develop during stage 3 . For assessment of the severity of LE, the ISL recommends the evaluation of volume differences between the upper extremities (mild LE $a<20 \%$ increase, moderate LE a $20-40 \%$ increase, and severe LE a $>40 \%$ increase), and also states that the most commonly used technique for volume measurement is circumferential measurement via a flexible non-stretch tape (6). The American Physical Therapy Association (APTA) uses arm circumference differences between the extremities to measure the severity of LE (mild LE $<3 \mathrm{~cm}$, moderate LE $3-5 \mathrm{~cm}$, and severe $\mathrm{LE}>5 \mathrm{~cm}$ ). Serial circumferential measurements should be performed and can be compared to baseline or unaffected arm (7). Although APTA recommends to measure each limb every $4 \mathrm{~cm}$, medial epicondyle or olecranon have been used as a landmark and standardized measurements have been applied in different studies (8-10). Treatment of LE is performed with conservative or surgical methods. Conservative treatment is a multimodal therapy that includes skin and nail care, exercises, and compression therapy. Self-monitoring, limb elevation, maintenance of ideal body weight, and the avoidance of infection, injury, tight fitting clothing, and blood pressure cuffing are general recommendations for patients with LE or for patients who are at risk for LE. The approach for LE therapy is based on the severity of the condition. For patients with a risk of developing LE (stage 0), general recommendations, protection of the extremity from harmful interventions and prevention of arm immobilization are suggested. Additional compression garments (CGs) can be used for patients with stage $1 \mathrm{LE}$. For moderate and severe (stage 2 and 3 ) LE, complete decongestive therapy (CDT), which is considered the gold standard for treatment against LE, should be performed. CDT is a two phase program. The first (treatment) phase consists of manual lymphatic drainage and multilayer bandaging in addition to general recommendations. The second (maintenance) phase aims to preserve gains from the first phase and includes CGs, exercises, and skin and nail care $(5,6)$. Intermittent pneumatic compression can also be used as a part of CDT (11). CGs are indicated to improve lymphatic flow, prevent lymphatic reaccumulation (CDT phase 2), maintain skin integrity, and avoid from potential trauma (12). LE can progress if the CGs are not correctly fitted or properly worn. The highest pressure that a patient can tolerate is the most likely to benefit them (6). It is recommended that CGs be replaced every three to six months or sooner if they lose their elasticity (13).

Kinesio taping (KT) is a relatively new treatment technique for LE. The technique and the material have been developed and introduced by Dr. Kenzo Kase. KT can decrease pain and relieve abnormal sensations. In addition, it can improve lymphatic and blood flow, and facilitate lymphatic drainage by microscopically lifting the skin (14-17). While some studies have evaluated the effectiveness of KT on BCAL, most have investigated advanced stages of LE or assessed if KT can be used as an alternative method to bandaging (18-20). In these studies, in addition to arm circumference, lymphedema related symptoms including pain, heaviness, tightness, and stiffness have been evaluated. KT has been shown to have positive effects on these measures. Researchers have observed that some patients encountered compliance difficulties with CGs, especially during the summer time. A number of patients were also unable to easily acquire CGs due to financial problems or time limitations. Therefore, this study aimed to evaluate 
KT's effectiveness in treating early stage BCAL in comparison to CGs.

\section{METHODS}

This study was approved by the local ethics committee of Marmara University. Oral and written informed consent were obtained from all participants and the study was conducted in accordance with the principles of the Declaration of Helsinki.

\section{Participants}

This study's participants included patients between 18-70-years old who had unilateral stage $1 \mathrm{BCAL}$ according to ISL, who had undergone at least 3 months of follow-up post breast surgery, and who had not received any LE treatment. Patients were excluded from the study if they had skin disease, infections, thrombophlebitis, and pregnancy, metastases in the lymph nodes, uncontrolled psychiatric and systemic diseases, sensorial or language problems, cognitive disorders, or were undergoing diuretic therapy.

\section{Study Design}

The study was designed as a prospective randomized single-blinded study. Patients with stage 1 BCAL were randomized into group I (KT) or group II (CG). Computer-generated random numbers in sealed opaque envelopes were used to allocate the interventions to the patients. An envelope containing the group allocation was opened by the independent physician for each patient. Patients were not available to be blind since the intervention was obvious. However, the outcome assessor was blinded to the allocation of the interventions. For sample size, estimation $\mathrm{G}$ power V3.1.9.2 was used. According to a similar study's data effect size was 1.0 to reach $80 \%$ power with a $5 \%$ type 1 error level, 13 patients were required in each group (18).

\section{Intervention}

Skin and nail care education, preventive measures (limb elevation, maintenance of ideal body weight, and avoidance of infection, injury, tight fitting clothing, or blood pressure cuffing), and exercises that included upper extremity ranges of motion, muscle pumping, and abdominal breathing were given to all patients. Exercises were prescribed for 20 minutes, twice a day. For group I, KT with a lymphatic correction technique was applied in three-four day intervals for four weeks. At the end of the fourth week, it was suggested that all the patients wear CGs. Application of the KT was performed on the arms and forearms with a 4 fan type tape. The anchor started with no tension, and the tails of the tape were applied to the anterior, medial, and posterior aspects of the forearms and arms with $15 \%$ tension (Fig. 1) $(21,22)$. For group II, the patients were treated daily 23 -hour CGs, education, preventive measures, and exercises. All patients were evaluated before the treatment (T0), immediate post treatment (T1), and three months after treatment (T2). Circumference differences were measured between the extremities with a nonelastic tape measurer at five levels that included the metacarpophalangeal (MCP) joints, the wrist, $15 \mathrm{~cm}$ distally from the medial epicondyle (ME), ME, and $15 \mathrm{~cm}$ proximally from the ME. Additionally, the shoulder range of motion (ROM) was measured with a goniometer, and pain, heaviness, and the sensation of tightness were assessed with a visual analog scale (VAS). A VAS change score of $-\mathbf{2 . 0}$ and a percent change score of $-33.0 \%$ were considered as appropriate cut-off points for clinically important improvement in pain scores based on the results of a previous study (23).

\section{Statistical Analysis}

Statistical analysis was performed with the SPSS 22.0 statistical package program. In addition to descriptive statistical methods (mean, frequency, percentage, and standard 


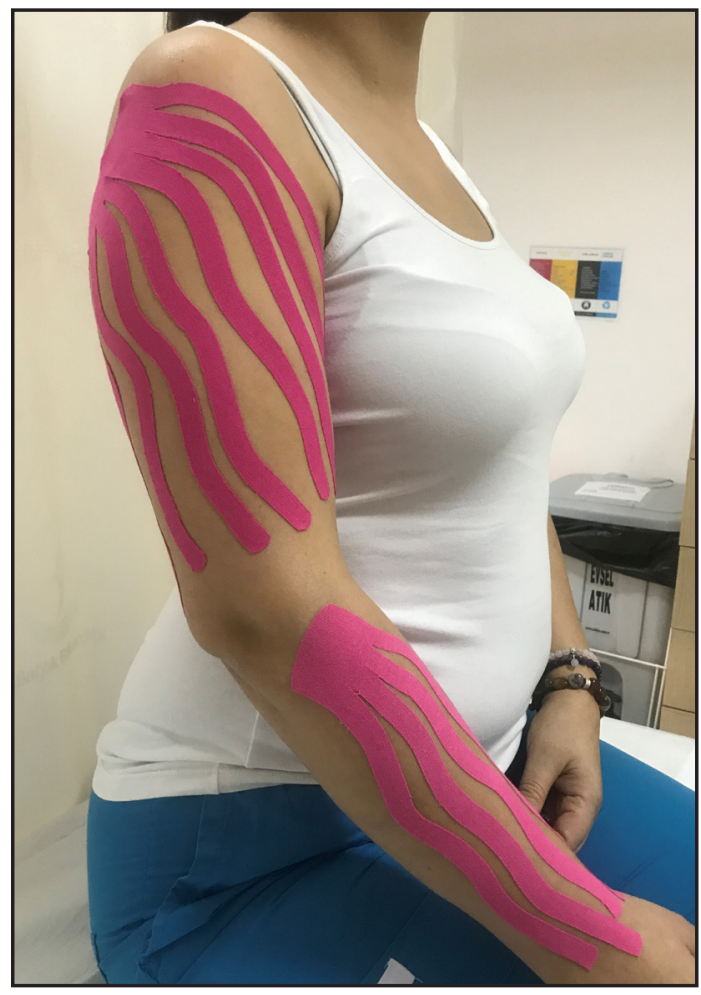

Fig. 1. Photograph of a patient demonstrating application of lymphatic correction KT for treatment utilized in this study.

deviation), the Shapiro-Wilk test was used to examine normal distribution parameters. Pearson's chi-squared test and Fisher's exact test were used to compare the qualitative data. The Student's t-test and the Mann-Whitney $\mathrm{U}$ test were used to compare the intergroup parameters. The Friedman test was used for repeated comparisons, and the Wilcoxon signed-rank test was performed with the Bonferroni correction for pairwise comparisons. The results were evaluated at a $95 \%$ confidence interval and a significance level of $\mathrm{p}<\mathbf{0 . 0 5}$.

\section{RESULTS}

Thirty-nine patients with stage $1 \mathrm{BCAL}$ were included in the study and randomized into either the KT $(n=19)$ or CG $(n=20)$ groups. Two patients in the KT group and one patient in the CG group were lost during follow-up, and one patient in the KT group had an allergy that resulted in their exclusion from the study. Thus, there were a total of $\mathbf{1 6}$ patients in the KT group and 19 patients in the CG group (Fig. 2). The demographic data and baseline clinical characteristics were similar for both groups $(\mathrm{p}<0.05)($ Table 1$)$.

\section{Intragroup Evaluations}

Both groups had reductions in all levels of arm circumference differences at immediate post-treatment and three months after treatment. When compared to the baseline, while there were significant differences at immediate post-treatment for all levels in the KT group, statistically significant differences were detected at immediate post-treatment in the wrist, $15 \mathrm{~cm}$ distally from the ME, and within the ME of the CG group. At three months after treatment, the significance difference was only present for the CG group in the measurement of $15 \mathrm{~cm}$ proximally from the ME (Table 2). Although shoulder ROM improved after treatment, significant differences in abduction were present in both groups and external rotation in CG group at immediate post-treatment. At the third month, significance was detected for abduction in CG group (Table 3). Pain, tightness, and heaviness scores significantly decreased for both groups at immediate post-treatment and third month (Table 4). At immediate post-treatment, 12 of 16 patients in KT group and 13 of 19 patients in CG group had clinically important improvement in pain scores. At third month, 9 of 16 patients in KT group and 14 of 19 patients in CG group had clinically important improvement in pain scores (Table 5).

\section{Intergroup Evaluations}

Arm measurements from all five points were similar at baseline and immediate post-treatment $(p>0.05)$. During the third month evaluations, although there was a significant difference in favor of the CG 


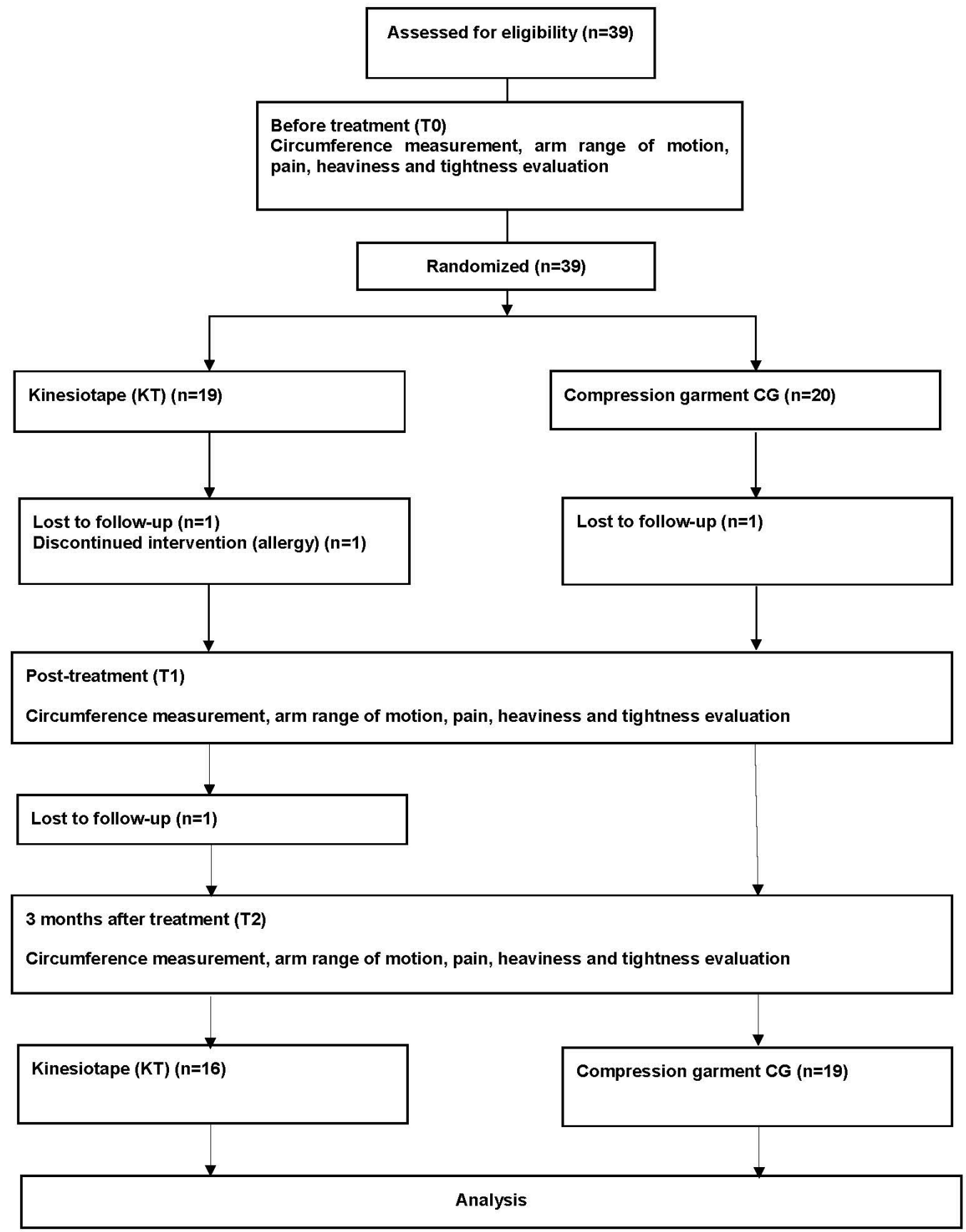

Fig. 2. Flow diagram of the study.

group at the MCP level ( $\mathrm{p}=0.032)$, the other measurements were similar for both groups (p>0.05) (Table 2). All shoulder movements were similar between the groups at baseline, immediate post-treatment (except external rotation), and the third month (Table 3). While all measurements for tightness and the sensation of heaviness were similar at immediate post-treatment and third month, the patients in the KT group had significantly lower mean 
TABLE 1

Demographic and Clinical Characteristics of Patients Enrolled in This Study

\begin{tabular}{|c|c|c|c|}
\hline & KT $(n=16)$ & CG $(n=19)$ & $\mathbf{P}$ \\
\hline Age $_{\text {mean }} \mathrm{SD}$ (years) & $50.56 \pm 6.45$ & $54.52 \pm 7.49$ & $0.107^{1}$ \\
\hline Height mean $\pm S D$ (centimeters) & $160.75 \pm 7.34$ & $161.05 \pm 4.02$ & $0.884^{1}$ \\
\hline Weight mean $\pm \mathrm{SD}$ (kilograms) & $73.62 \pm 14.21$ & $76.10 \pm 12.61$ & $0.589^{1}$ \\
\hline $\mathrm{BMI}_{\text {mean } \pm \mathrm{SD}\left(\mathrm{kg} / \mathrm{m}^{2}\right)}$ & $29.58 \pm 6.29$ & $29.28 \pm 4.46$ & $0.873^{1}$ \\
\hline Lymphedema duration mean $\pm \mathrm{SD}$ (months) & $9.68 \pm 2.60$ & $8.68 \pm 3.55$ & $0.344^{1}$ \\
\hline \multicolumn{4}{|l|}{ Lymphedema side $\mathrm{n}(\%)$} \\
\hline Right & 8 & 11 & \multirow{2}{*}{$0.640^{2}$} \\
\hline Left & 8 & 8 & \\
\hline Chemotherapy (Yes/No) & $14 / 2$ & $17 / 2$ & $0.855^{2}$ \\
\hline Radiotherapy (Yes/No) & $13 / 3$ & $15 / 4$ & $0.865^{2}$ \\
\hline Axillary lymph node dissection & 16 & 19 & - \\
\hline Duration after surgery mean $\pm \mathrm{SD}$ (months) & $17.50 \pm 5.95$ & $17.15 \pm 9.58$ & $0.898^{1}$ \\
\hline \multicolumn{4}{|l|}{ Surgery method } \\
\hline Modified radical mastectomy $\mathrm{n}(\%)$ & $12(75 \%)$ & $16(84.2 \%)$ & \\
\hline Breast-conserving surgery $\mathrm{n}(\%)$ & $4(25 \%)$ & $3(15.8 \%)$ & \\
\hline
\end{tabular}

${ }^{1}$ student-t test; ${ }^{2}$ Chi-square test

pain scores than patients in the CG group at immediate post-treatment (Table 4). The percentage of patients who had clinically important improvement in pain scores were similar for both groups at immediate post-treatment and third month (Table 5).

\section{DISCUSSION}

This study aimed to evaluate the effectiveness of KT by comparing it to CGs during early stage BCAL treatment. Both groups were found to be effective in terms of differences in arm circumference and all patients also showed significant improvement in pain and the sensations of tightness and heaviness.

$\mathrm{KT}$ is used to treat musculoskeletal pathologies, neurological disorders, sport injuries, and LE (17,24-29). It can improve blood flow and lymphatic flow by lifting the skin at the microscopic level and reducing pressure. Thus, it also can decrease pain and inflammation and permits joint ROM (30). Although most studies have evaluated the efficacy of KT for treatment of LE in BCAL patients, a few have determined its effectiveness on lower extremity LE or chronic venous insufficiency (18-20,31-33). In a randomized single-blinded study that assessed the efficacy of KT in the treatment of grade 2 and grade 3 $\mathrm{BCAL}$, it was determined that $\mathrm{KT}$ cannot be used as an alternative to multilayer bandaging (19). According to the ISL consensus on advanced stage LE, CDT is the gold standard for treatment and multilayer bandaging is a component of CDT (6). When Pekyavas et al. randomized patients into three groups that included a CDT group, a CDT with KT group, and a CDT with KT but no bandaging group, they concluded that KT had positive effects on LE symptoms and brought longevity to the effects of the LE treatment (20). The current study included patients with stage $1 \mathrm{BCAL}$ who had not received any LE treatment. The results showed that $\mathrm{KT}$ was not inferior to CGs. In another study that examined the effects of KT on stage 1 and 2 BCAL, KT positively impacted upper limb motion and grip strength (34). The present study determined results that were consistent with these findings, observing improved upper extremity ROM in both the KT and CG groups. There were no significant differences in these results between either group. When Tantawy et al. compared exercise with a combination of KT and exercise to investigate their influence on ROM and shoulder disability in post-mastectomy patients, they determined that KT 


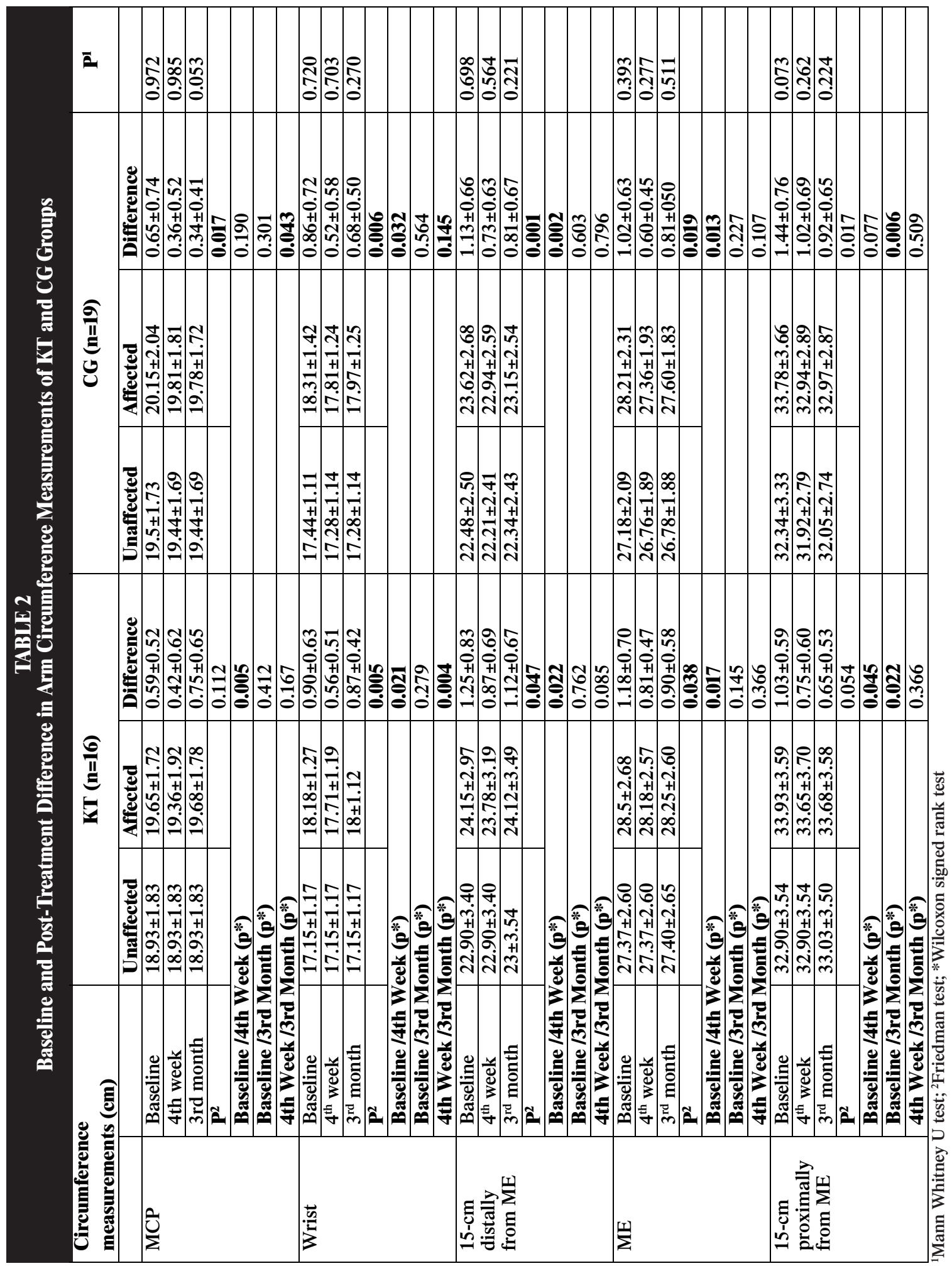


TABLE 3

Baseline and Post-Treatment Differences in Shoulder Range of Movement of KT and CG Groups

\begin{tabular}{|c|c|c|c|c|}
\hline \multicolumn{2}{|c|}{ Shoulder ROM } & KT $(n=16)$ & CG $(n=19)$ & $\mathbf{P} \mathbf{T}$ \\
\hline \multirow[t]{7}{*}{ Abduction } & Baseline & $157.50 \pm 23.52$ & $164.21 \pm 18.94$ & 0.481 \\
\hline & $4^{\text {th }}$ week & $163.75 \pm 15.43$ & $167.89 \pm 17.50$ & 0.259 \\
\hline & $3^{\text {rd }}$ month & $160 \pm 23.66$ & $167.89 \pm 17.50$ & 0.088 \\
\hline & $\mathbf{P}^{2}$ & $\mathbf{0 . 0 5}$ & 0.002 & \\
\hline & Baseline /4th Week (p*) & 0.041 & 0.020 & \\
\hline & Baseline /3rd Month (p*) & 0.344 & $\mathbf{0 . 0 2 0}$ & \\
\hline & 4th Week /3rd Month (p*) & 0.713 & 1.00 & \\
\hline \multirow[t]{7}{*}{ Adduction } & Baseline & $38.12 \pm 4.03$ & $38.42 \pm 6.88$ & 0.254 \\
\hline & $4^{\text {th }}$ week & $39.37 \pm 2.50$ & $37.36 \pm 7.33$ & 0.368 \\
\hline & $3^{\text {rd }}$ month & $38.75 \pm 3.41$ & $37.89 \pm 7.13$ & 0.905 \\
\hline & $\mathbf{P}^{2}$ & 0.368 & 0.368 & \\
\hline & Baseline /4th Week (p*) & 0.157 & 0.157 & \\
\hline & Baseline /3rd Month (p*) & 0.317 & 0.317 & \\
\hline & 4th Week /3rd Month (p*) & 0.564 & 0.564 & \\
\hline \multirow[t]{7}{*}{ Flexion } & Baseline & $159.37 \pm 26.44$ & $166.31 \pm 10.65$ & 0.983 \\
\hline & $4^{\text {th }}$ week & $163.75 \pm 18.21$ & $168.94 \pm 13.28$ & 0.367 \\
\hline & $3^{\text {rd }}$ month & $165 \pm 16.32$ & $167.89 \pm 17.50$ & 0.409 \\
\hline & $\mathbf{P}^{2}$ & 0.024 & 0.076 & \\
\hline & Baseline /4th Week (p*) & 0.066 & 0.096 & \\
\hline & Baseline /3rd Month (p*) & 0.066 & 0.332 & \\
\hline & 4th Week /3rd Month (p*) & 0.157 & 0.317 & \\
\hline \multirow[t]{7}{*}{ Extension } & Baseline & $36.87 \pm 7.93$ & $35.78 \pm 6.06$ & 0.453 \\
\hline & $4^{\text {th }}$ week & $40.00 \pm 6.32$ & $36.84 \pm 5.82$ & 0.190 \\
\hline & $3^{\text {rd }}$ month & $38.12 \pm 4.03$ & $37.36 \pm 5.61$ & 0.812 \\
\hline & $\mathbf{P}^{2}$ & 0.092 & 0.247 & \\
\hline & Baseline /4th Week (p*) & 0.059 & 0.157 & \\
\hline & Baseline /3rd Month (p*) & 0.577 & 0.083 & \\
\hline & 4th Week /3rd Month (p*) & 0.317 & 0.655 & \\
\hline \multirow{7}{*}{$\begin{array}{l}\text { Internal } \\
\text { Rotation }\end{array}$} & Baseline & $73.75 \pm 14.54$ & $75.78 \pm 14.26$ & 0.572 \\
\hline & $4^{\text {th }}$ week & $76.87 \pm 9.46$ & $76.31 \pm 14.22$ & 0.875 \\
\hline & $3^{\text {rd }}$ month & $79.37 \pm 9.28$ & $75.78 \pm 13.87$ & 0.191 \\
\hline & $\mathbf{P}^{2}$ & 0.015 & 0.607 & \\
\hline & Baseline /4th Week (p*) & 0.059 & 0.317 & \\
\hline & Baseline /3rd Month (p*) & 0.039 & 1.00 & \\
\hline & 4th Week /3rd Month (p*) & 0.180 & 0.317 & \\
\hline \multirow{7}{*}{$\begin{array}{l}\text { External } \\
\text { Rotation }\end{array}$} & Baseline & $75.00 \pm 15.05$ & $82.10 \pm 5.35$ & 0.091 \\
\hline & $4^{\text {th }}$ week & $77.50 \pm 10.00$ & $85.26 \pm 6.11$ & 0.006 \\
\hline & $3^{\text {rd }}$ month & $78.12 \pm 10.46$ & $83.68 \pm 5.97$ & 0.082 \\
\hline & $\mathbf{P}^{2}$ & 0.061 & 0.021 & \\
\hline & Baseline /4th Week (p*) & 0.102 & 0.014 & \\
\hline & Baseline /3rd Month (p*) & 0.102 & 0.180 & \\
\hline & 4th Week /3rd Month (p*) & 0.317 & 0.083 & \\
\hline
\end{tabular}

${ }^{1}$ Mann Whitney U test; ${ }^{2}$ Friedman test; *Wilcoxon signed rank test 


\begin{tabular}{|c|c|c|c|c|}
\hline \multicolumn{5}{|c|}{$\begin{array}{l}\text { TABLE } 4 \\
\text { Baseline and Post-Treatment Differences in Pain, Tightness, and Heaviness Scores of } \\
\text { KT and CG Groups }\end{array}$} \\
\hline VAS & & KT $(n=16)$ & CG $(n=19)$ & $\mathbf{p}$ \\
\hline \multirow{7}{*}{ Pain } & Baseline & $2.87 \pm 1.54$ & $2.73 \pm 0.99$ & 0.412 \\
\hline & 4th week & $0.81 \pm 1.04$ & $1.73 \pm 0.65$ & 0.006 \\
\hline & 3rd month & $1.68 \pm 1.07$ & $1.31 \pm 1.24$ & 0.364 \\
\hline & $\mathbf{P}^{2}$ & $\mathbf{0 . 0 0 0 1}$ & 0.0001 & \\
\hline & Baseline /4th Week (p*) & 0.002 & $\mathbf{0 . 0 0 2}$ & \\
\hline & Baseline /3rd Month (p*) & 0.002 & 0.001 & \\
\hline & 4th Week /3rd Month (p*) & 0.006 & $\mathbf{0 . 1 3 7}$ & \\
\hline \multirow{7}{*}{ Tightness } & Baseline & $3.12 \pm 1.54$ & $2.36 \pm 0.83$ & 0.058 \\
\hline & 4th week & $1.18 \pm 1.42$ & $1.68 \pm 0.82$ & 0.181 \\
\hline & 3rd month & $1.87 \pm 0.95$ & $1.36 \pm 1.34$ & 0.216 \\
\hline & $\mathbf{P}^{2}$ & 0.003 & 0.007 & \\
\hline & Baseline /4th Week (p*) & 0.002 & $\mathbf{0 . 0 3 8}$ & \\
\hline & Baseline /3rd Month (p*) & 0.010 & 0.010 & \\
\hline & 4th Week /3rd Month (p*) & 0.060 & 0.281 & \\
\hline \multirow{7}{*}{ Heaviness } & Baseline & $3.12 \pm 1.08$ & $2.84 \pm 1.06$ & 0.079 \\
\hline & 4th week & $1.75 \pm 1.61$ & $1.47 \pm 0.90$ & 0.657 \\
\hline & 3rd month & $1.75 \pm 1.18$ & $1.21 \pm 1.18$ & 0.184 \\
\hline & $\mathbf{P}^{2}$ & 0.004 & 0.0001 & \\
\hline & Baseline /4th Week (p*) & 0.008 & 0.001 & \\
\hline & Baseline /3rd Month (p*) & $\mathbf{0 . 0 0 2}$ & 0.0001 & \\
\hline & 4th Week /3rd Month (p*) & 1.00 & 0.290 & \\
\hline
\end{tabular}

${ }^{1}$ Mann Whitney U test; ${ }^{2}$ Friedman test; *Wilcoxon signed rank test

\section{TABLE 5}

Patients with and without Clinically Improvement Change in Pain Scores of KT and CG Groups

\begin{tabular}{cllllll} 
& & $\begin{array}{l}\text { KT } \\
(\mathrm{n}=16)\end{array}$ & $\%$ & $\begin{array}{l}\text { CG } \\
(\mathrm{n}=19)\end{array}$ & $\%$ & $\mathrm{p}$ \\
\hline Clinically important change & & & & & & \\
Baseline/4 & & & & \\
& yes & 12 & $75 \%$ & 13 & $68.4 \%$ & $0.723^{1}$ \\
Baseline $/ 3^{\text {rd }}$ month & no & 4 & $25 \%$ & 6 & $31.6 \%$ & \\
& yes & 9 & $56.3 \%$ & 14 & $73.7 \%$ & $0.279^{2}$ \\
\hline
\end{tabular}

${ }^{1}$ Fischer exact test; ${ }^{2}$ Chi-square test

significantly improved pain intensity, disability scores, and shoulder ROM (31). According to a study that investigated if $\mathrm{KT}$ could replace bandaging in decongestive lymphatic therapy (DLT) for BCAL, LE symptoms that had been evaluated with a VAS and treated with both standard DLT and modified DLT (with KT) showed improvement in the symptoms of fullness, tightness, and discomfort. In the modified DLT group (with KT), the symp- toms of pain, hardness, and tingling showed significant improvement, as well. As a result, the researchers concluded that KT could replace bandaging in patients who had poor compliance with normal bandage use after one month of intervention (18). Similarly, the VAS from the current study showed significant reductions in pain, tightness, and heaviness in both groups. 
This study was limited in that it did not test the combined treatment of KT with CGs. This testing was not planned, as it was impossible to dress the CGs over the KT. In addition, because CDT is the gold standard for the treatment of BCAL, stage $1 \mathrm{BCAL}$ was the only stage of the condition that was investigated, as any treatment protocol without lymphatic drainage would have proven unethical. Moreover, since other clinicians have already studied how KT can impact CDT, the current research aimed to assess if $\mathrm{KT}$ could be used as an alternative to CGs during early stage BCAL treatment.

To the best of our knowledge, this is the first study to compare KT with CGs and to evaluate treatment on stage $1 \mathrm{BCAL}$. In conclusion, KT is not inferior to CGs during the early stages of BCAL treatment. In addition, KT may also benefit patients who face delays in obtaining CGs, those who have a low compliance with the treatment, or in those who have difficulties wearing CGs.

\section{CONFLICT OF INTEREST AND DISCLO- SURE}

All authors declare no competing financial interests exist.

\section{REFERENCES}

1. DiSipio, T, S Rye, B Newman, et al: Incidence of unilateral arm lymphoedema after breast cancer: A systematic review and meta-analysis. Lancet Oncol. 14 (2013), 500-515.

2. Armer, JM, KV Ballman, L McCall, et al: Factors associated with lymphedema in women with node-positive breast cancer treated with neoadjuvant chemotherapy and axillary dissection. JAMA Surg. 154 (2019), 800-809.

3. Cariati, M, S Bains, M Grootendorst, et al: Adjuvant taxanes and the development of breast cancer-related arm lymphoedema. Brit. J. Surg. 102 (2015), 1071-1078.

4. Gross, JP, S Sachdev, IB Helenowski, et al: Radiation therapy field design and lymphedema risk after regional nodal irradiation for breast Cancer. Int. J. Radiat. Oncol. 102 (2018), 71-78.

5. Lawenda, BD, TE Mondry, PA Johnstone: Lymphedema: A primer on the identification and management of a chronic condition in oncologic treatment. CA-Cancer J. Clin. 59 (2009), 8-24.

6. The Diagnosis and Treatment of Peripheral Lymphedema: 2016 Consensus Document of the International Society of Lymphology. Lymphology 49 (2016), 170-184.

7. Bernas, MJ, RL Askew, JM Armer, et al: Lymphedema: How do we diagnose and reduce the risk of this dreaded complication of breast cancer treatment? Curr. Breast Cancer Rep. 2 (2010), 53-58.

8. McLaughlin, SA, MJ Wright, KT Morris, et al: Prevalence of lymphedema in women with breast cancer 5 years after sentinel lymph node biopsy or axillary dissection: Objective measurements. J. Clin. Oncol. 26 (2008), 5213.

9. Kozanoglu, E, S Basaran, S Paydas, et al: Efficacy of pneumatic compression and low-level laser therapy in the treatment of postmastectomy lymphoedema: A randomized controlled trial. Clin. Rehabil. 23 (2009), 117-124.

10. Lucci, A, LM McCall, PD Beitsch, et al: Surgical complications associated with sentinel lymph node dissection (SLND) plus axillary lymph node dissection compared with SLND alone in the American College of Surgeons Oncology Group Trial Z0011. J. Clin. Oncol. 25 (2007), 3657-3663.

11. Sanal-Toprak C, T Ozsoy-Unubolo, Y Bahar-Ozdemir, et al: The efficacy of intermittent pneumatic compression as a substitute for manual lymphatic drainage in complete decongestive therapy in the treatment of breast cancer related lymphedema. Lymphology 52 (2019), 82-91.

12. Cheville, AL: Current and future trends in lymphedema management: Implications for women's health. Phys. Med. Reh. Clin N. 18 (2007), 539-553.

13. Poage, E, M Singer, J Armer, et al: Demystifying lymphedema: Development of the lymphedema putting evidence into practice card. Clin. J. Oncol. Nurs. 12 (2008), 951-964.

14. Aguilar-Ferrándiz, ME, AM Castro-Sánchez, GA Matarán-Peñarrocha, et al: Effects of kinesio taping on venous symptoms, bioelectrical activity of the gastrocnemius muscle, range of ankle motion, and quality of life in postmenopausal women with chronic venous insufficiency: A randomized controlled trial. Arch. Phys. Med. Rehab. 94 (2013), 2315-2328.

15. Aguilar-Ferrándiz, ME, AM Castro-Sánchez, GA Matarán-Peñarrocha, et al: A randomized controlled trial of a mixed Kinesio taping-compression technique on venous symptoms, pain, peripheral venous flow, clinical severity 
and overall health status in postmenopausal women with chronic venous insufficiency. Clin. Rehab. 28 (2014), 69-81.

16. Craighead, DH, SW Shank, KM Volz, et al: Kinesiology tape modestly increases skin blood flow regardless of tape application technique. J. Perform. Health Res. (2017), 1787.

17. Kasawara, KT, KMR Mapa, V Ferreira, et al: Effects of Kinesio Taping on breast cancer-related lymphedema: A meta-analysis in clinical trials. Physiother. Theor. Pr. 34 (2018), 337-345.

18. Tsai, H-J, H-C Hung, J-L Yang, et al: Could Kinesio tape replace the bandage in decongestive lymphatic therapy for breast-cancer-related lymphedema? A pilot study. Suppor. Care Cancer 17 (2009), 1353.

19. Smykla, A, K Walewicz, R Trybulski, et al: Effect of Kinesiology Taping on breast cancer-related lymphedema: A randomized single-blind controlled pilot study. BioMed Res. Int. 2013:767106. doi: 10.1155/2013/767106. Epub 2013 Nov 27.

20. Pekyavaş NÖ, VB Tunay, T Akbayrak, et al: Complex decongestive therapy and taping for patients with postmastectomy lymphedema: A randomized controlled study. Eur. J. Oncol. Nurs. 18 (2014), 585-590.

21. Malicka, I, A Rosseger, J Hanuszkiewicz, et al: Kinesiology Taping reduces lymphedema of the upper extremity in women after breast cancer treatment: A pilot study. Przeglad Menopauzalny 13 (2014), 221-226.

22. Taradaj, J, T Halski, M Zduńczyk, et al: Evaluation of the effectiveness of kinesio taping application in a patient with secondary lymphedema in breast cancer: A case report. Przeglad Menopauzalny 13 (2014), 73.

23. Salaffi, F, A Stancati, CA Silvestri, et al: Minimal clinically important changes in chronic musculoskeletal pain intensity measured on a numerical rating scale. Eur. J. Pain 8 (2004), 283-291.

24. Morris, D, D Jones, H Ryan, et al: The clinical effects of Kinesio ${ }^{\circledR}$ Tex taping: A systematic review. Physiother. Theor. Pr. 29 (2013), 259270.

25. Giray, E, DK Bingul, G Akyuz: The effectiveness of kinesiotaping, sham taping or exercises only in treatment of lateral epicondylitis: A randomized controlled study. PM.R. 7 (2019), 681-693.

26. Mansiz Kaplan, B, G Akyuz, S Kokar, et al: Comparison of the effectiveness of orthotic intervention, kinesiotaping, and paraffin treatments in patients with carpal tunnel syndrome: A single-blind and randomized controlled study. J. Hand Ther. 32 (2019), 297-304.
27. Yam, TTT, MS Wong, SSM Fong: Effect of Kinesio taping on electromyographic activity of leg muscles during gait in children with developmental coordination disorder: A randomized controlled trial. Medicine 98 (2019), e14423.

28. Dos Santos, AN, N Rocha: Immediate effect of kinesio taping on knee extensor torque of children with Cerebral Palsy: Three case reports. Neurorehabilitation 43 (2018), 519-523.

29. Yen, SC, E Folmar, KA Friend, et al: Effects of kinesiotaping and athletic taping on ankle kinematics during walking in individuals with chronic ankle instability: A pilot study. Gait Posture 66 (2018), 118-123.

30. Mostafavifar, M, J Wertz, J Borchers: A systematic review of the effectiveness of kinesio taping for musculoskeletal injury. Phys. Sportsmed. 40 (2012), 33-40.

31. Tantawy, SA, DM Kamel: The effect of kinesio taping with exercise compared with exercise alone on pain, range of motion, and disability of the shoulder in postmastectomy females: A randomized control trial. J. Phys. Ther. Sci. 28 (2016), 3300-3305.

32. Aguilar-Ferrándiz, ME, C Moreno-Lorenzo, GA Matarán-Peñarrocha, et al: Effect of a mixed kinesio taping-compression technique on quality of life and clinical and gait parameters in postmenopausal women with chronic venous insufficiency: Double-blinded, randomized controlled trial. Arch. Phys. Med. Rehab. 95 (2014), 1229-1239.

33. Gerasimenko, MY, TA Knyazeva, V T, et al: [The application of the method of kinesio-taping technique for the combined non-pharmacological rehabilitation of the patients presenting with lymphedema of the lower extremities]. Vopr. Kurortol. Fizioter. Lech. Fiz. Kult. 92 (2015), 22-27.

34. Pop, TB, B Karczmarek-Borowska, M Tymczak, et al: The influence of Kinesiology Taping on the reduction of lymphoedema among women after mastectomy-preliminary study. Contemp. Oncol. 18 (2014), 124.

\author{
Dr. Canan Sanal-Toprak \\ Assistant Professor \\ Marmara Universitesi Eğitim araştırma \\ Hastanesi Fiziksel Tip ve Rehabilitasyon \\ Anabilim Dalı Pendik/Istanbul TURKEY \\ Tel: 90(533)1381032 \\ Fax: 90(216)6254545 \\ e-mail: canansanal@hotmail.com
}

